ANNALES

POLONICI MATHEMATICI

LXX (1998)

\title{
Analytic hypoellipticity for sums of squares of vector fields
}

\author{
by A. Alexandrou Himonas (Notre Dame, Ind.)
}

\begin{abstract}
We discuss the open problem of analytic hypoellipticity for sums of squares of vector fields, including some recent partial results and a conjecture of Treves.
\end{abstract}

1. Introduction. Let $\mathcal{M}^{n}$ be an analytic manifold and $X=\left\{X_{1}, \ldots\right.$ $\left.\ldots, X_{\nu}\right\}$ be a collection of real vector fields with coefficients in $\mathcal{C}^{\omega}\left(\mathcal{M}^{n}\right)$, the real analytic functions on $\mathcal{M}^{n}$. In this paper, $\mathcal{M}^{n}$ will be an open set $\Omega$ in $\mathbb{R}^{n}$, or the $n$-dimensional torus, $\mathbb{T}^{n}$. The sum of squares operator or sublaplacian associated with the vector fields $X$ is the second order partial differential operator defined by

$$
P=\Delta_{X} \doteq X_{1}^{2}+\ldots+X_{\nu}^{2} .
$$

We recall that an operator $P$ is called analytic hypoelliptic in $\mathcal{M}^{n}$ if for every open subset $U$ of $\mathcal{M}^{n}$ we have

$$
P u=f, u \in \mathcal{D}^{\prime}(U), f \in C^{\omega}(U) \Rightarrow u \in \mathcal{C}^{\omega}(U) .
$$

$P$ is called hypoelliptic in $\mathcal{M}^{n}$ if (1.2) holds with $\mathcal{C}^{\omega}$ replaced with $\mathcal{C}^{\infty}$, and globally analytic hypoelliptic in $\mathcal{M}^{n}$ if (1.2) holds for $U=\mathcal{M}^{n}$. The well known Laplacian in $\mathbb{R}^{n}$ is the typical example of an analytic hypoelliptic operator. If $\nu<n$ then $P=\left(\partial / \partial x_{1}\right)^{2}+\ldots+\left(\partial / \partial x_{\nu}\right)^{2}$ is not hypoelliptic, nor analytic hypoelliptic in $\mathbb{R}^{n}$ since there are "missing" directions. The vector fields $X$ are said to satisfy the bracket condition at a point $x \in \mathcal{M}^{n}$ if the Lie algebra generated by them spans the tangent space to $\mathcal{M}^{n}$ at $x$. Moreover, the length $k=k(x) \geq 1$ of the longest bracket needed to generate the tangent space at $x$ is called the type of the point $x$. Here, each $X_{j}$ is considered to be a bracket of length $1,\left[X_{j}, X_{l}\right]$ is a bracket of length 2 , and so on. For example, if $k=1$ for all $x$ in $\mathcal{M}^{n}$ then the operator $\Delta_{X}$ is elliptic and therefore hypoelliptic, and analytic hypoelliptic.

1991 Mathematics Subject Classification: Primary 35H05.

Key words and phrases: analytic hypoellipticity, sum of squares of vector fields, eigenvalue, bracket condition, characteristic set, symplectic, torus.

Partially supported by NSF. 
The following theorem follows from the celebrated theorem of Hörmander [Ho] (see also Kohn [K], Oleĭnik and Radkevich [OR2], Rothschild and Stein $[R S])$ and a result of Derridj in $[D]$.

Theorem 1.1. The operator $\Delta_{X}$ is hypoelliptic in $\mathcal{M}^{n}$ if and only if the bracket condition holds at every point $x \in \mathcal{M}^{n}$.

Therefore, in the case of analytic coefficients the hypoellipticity of the operator $\Delta_{X}$ is equivalent to the bracket condition. In the case of $\mathcal{C}^{\infty}$ coefficients the bracket condition implies the hypoellipticity of $\Delta_{X}$ (see [Ho]). However, there are operators $\Delta_{X}$ which are hypoelliptic and the bracket condition does not hold (see Fediu [F], Kusuoka and Strook [KS], Bell and Mohammed [BM]).

Here we only consider real-analytic vector fields $X$ and discuss the problem of local and global analytic hypoellipticity. By the above theorem we must assume that the bracket condition holds in $\mathcal{M}^{n}$.

2. Local analytic hypoellipticity. In 1972 Baouendi and Goulaouic [BG] gave the first example of an operator $\Delta_{X}$ which satisfies the bracket condition and yet is not analytic hypoelliptic. They proved that the operator

$$
\Delta_{X}=\left(\frac{\partial}{\partial x_{1}}\right)^{2}+\left(\frac{\partial}{\partial x_{2}}\right)^{2}+\left(x_{1} \frac{\partial}{\partial x_{3}}\right)^{2}
$$

is not analytic hypoelliptic. This operator is elliptic except at the points on the plane $x_{1}=0$, where $\left[\partial / \partial x_{1}, x_{1} \partial / \partial x_{3}\right]=\partial / \partial x_{3}$, and therefore the bracket condition holds. This operator was the starting point for many other counterexamples and partial positive results on analytic hypoellipticity by different authors trying to understand the following problem.

Open Problem 1. Assume that the bracket condition holds. What is a necessary and sufficient condition for the analytic hypoellipticity of $\Delta_{X}$ ?

Let

$$
P(x, \xi)=X_{1}^{2}(x, \xi)+\ldots+X_{\nu}^{2}(x, \xi)
$$

be the principal symbol of $\Delta_{X}$ and

$$
\Sigma=\left\{X_{1}(x, \xi)=\ldots=X_{\nu}(x, \xi)\right\}
$$

be its characteristic set. The following theorem of Tartakoff [Ta1] and Treves [Tr1] provides a sufficient condition in terms of the geometry of $\Sigma$.

THeOREM 2.1. Let $\Omega$ be an open set in $\mathbb{R}^{n}$. The operator $\Delta_{X}$ is analytic hypoelliptic in $\Omega$ if:

(a) The characteristic set $\Sigma$ is an analytic symplectic submanifold of $T^{*}(\Omega)-0$.

(b) The symbol $P(x, \xi)$ vanishes exactly to order two on $\Sigma$. 
This theorem has been generalized by Métivier [Met1] and Sjöstrand [S] to more general operators with multiple characteristics, symplectic set $\Sigma$, and higher but fixed order of vanishing of the symbol on $\Sigma$. We recall that $\Sigma$ is called symplectic if the restriction of the fundamental symplectic form

$$
\sigma=\sum_{j=1}^{n} d \xi_{j} \wedge d x_{j}
$$

to $T \Sigma$ is non-degenerate.

The symplecticity of $\Sigma$ does not allow the existence of Treves curves in it. We recall that a non-constant curve $\alpha(t)$ inside the characteristic set $\Sigma$ is called a Treves curve if $d \alpha / d t$ is orthogonal to $T \Sigma$ with respect to $\sigma$ at every point of $\alpha$. That is,

$$
\sigma(d \alpha / d t, \Theta)=0, \quad \forall \Theta \in T \Sigma, \text { at every point on } \alpha .
$$

In the case of the operator (2.1) the principal symbol is $P(x, \xi)=\xi_{1}^{2}+\xi_{2}^{2}+$ $x_{1}^{2} \xi_{3}^{2}$, the characteristic set is $\Sigma=\left\{x_{1}=\xi_{1}=\xi_{2}=0\right\}$, and the $x_{2}$-lines inside $\Sigma$ are Treves curves. In [Tr3] Treves conjectured that the existence of such curves inside $\Sigma$ should imply the non-hypoellipticity of $\Delta_{X}$. More precisely, he proposed the following conjecture.

CONJECture 1. A necessary condition for $\Delta_{X}$ to be analytic hypoelliptic is that its characteristic set contains no Treves curves.

This conjecture still remains unsettled. However, the next result by Hanges and Himonas [HH4] shows that the condition in Conjecture 1 is not sufficient.

TheOREM 2.2. Let $k$ be an odd positive integer. Then for the operator $P_{k}$ in $\mathbb{R}^{3}$ defined by

$$
P_{k}=\left(\frac{\partial}{\partial x_{1}}\right)^{2}+\left(x_{1}^{(k-1) / 2} \frac{\partial}{\partial x_{2}}\right)^{2}+\left(x_{1}^{k} \frac{\partial}{\partial x_{3}}\right)^{2}
$$

one can construct non-analytic solutions to the equation $P_{k} u=0$ near $x_{1}=0$.

Observe that for $k=1$ we obtain the Baouendi-Goulaouic operator which has non-symplectic characteristic set containing Treves curves, while for $k=3,5,7, \ldots$ the characteristic set is $\Sigma=\left\{x_{1}=\xi_{1}=0\right\}$, which is symplectic and thus contains no Treves curves. Therefore, the absence of Treves curves does not imply analytic hypoellipticity.

The operators $P_{k}$ in (2.5) form a subclass of the following class of operators:

$$
P=\left(\frac{\partial}{\partial x_{1}}\right)^{2}+\left(x_{1}^{m} \frac{\partial}{\partial x_{2}}\right)^{2}+\left(x_{1}^{k} \frac{\partial}{\partial x_{3}}\right)^{2}
$$


where $m, k$ are non-negative integers with $0 \leq m \leq k$, studied by Oleŭnik and Radkevich [OR1]. They proved that $P$ is analytic hypoelliptic if and only if $m=k$. The non-hypoellipticity was proved by indirect methods.

Here we outline an explicit and elementary construction of singular solutions to $P_{k} u=0$ presented in [HH4]. By using separation of variables we find that

$$
u(x)=\int_{0}^{\infty} e^{i \varrho^{k+1} x_{3}} e^{\sqrt{\mu} x_{2} \varrho^{(k+1) / 2}} A\left(\varrho x_{1}\right) w(\varrho) d \varrho
$$

is a formal solution to $P_{k} u=0$ if $A$ satisfies the eigenvalue problem

$$
\left(-\frac{d^{2}}{d t^{2}}+t^{2 k}\right) A(t)=\mu t^{k-1} A(t) .
$$

For $u$ to be well defined and non-trivial we require

$$
A \in \mathcal{S}(\mathbb{R})-\{0\},
$$

and

Then by letting

$$
w(\varrho)=e^{-\varrho^{(k+1) / 2}} .
$$

equation (2.8) takes the form

$$
-B^{\prime \prime}+2 t^{k} B^{\prime}-\mu t^{k-1} B=0 .
$$

If $k=1$ then (2.11) is the Hermite equation. To solve (2.11) we proceed as for the Hermite equation. We look for solutions in the form of a power series $B(x)=\sum_{j=0}^{\infty} b_{j} x^{j}$ and we find that if $\mu$ is in the set

(2.12) $\quad M=\{\mu: \mu=2 j(k+1)+k$ or $\mu=2 j(k+1)+k+2, j=0,1,2, \ldots\}$, then $B$ is a polynomial $B_{\mu}$. In addition we show that only for $\mu \in M$ do we have $A_{\mu}(t)=B_{\mu}(t) e^{-\frac{1}{k+1} t^{k+1}} \in \mathcal{S}(\mathbb{R})$. Therefore, for each $\mu \in M$ we have a solution

$$
u_{\mu}(x)=\int_{0}^{\infty} e^{i \varrho^{k+1} x_{3}+\left(\sqrt{\mu} x_{2}-1\right) \varrho^{(k+1) / 2}} A_{\mu}\left(\varrho x_{1}\right) d \varrho
$$

to $P_{k} u_{\mu}=0$ which is well defined for $\left\{\left|x_{2}\right|<1 / \sqrt{\mu}\right\}$. It is easy to check that $u_{\mu}$ is $\mathcal{C}^{\infty}$. To check that $u_{\mu}$ is not analytic at $x=0$ we assume $A_{\mu}(0) \neq 0$ (otherwise $A_{\mu}^{\prime}(0) \neq 0$ ) and obtain

$$
\left|\partial_{x_{3}}^{j} u_{\mu}(0)\right|=\left|A_{\mu}(0) \int_{0}^{\infty} \varrho^{j(k+1)} e^{-\varrho^{(k+1) / 2}} d \varrho\right| \geq C 2^{j}(2 j) ! .
$$

This shows that $u_{\mu}$ is not analytic near $0 \in \mathbb{R}^{3}$. In fact $u_{\mu}$ is in Gevrey class 2. It can be shown (see Christ [Ch5]) that this is optimal. 
If $\mu=k$ then (2.13) gives the following explicit solution to $P_{k} u=0$ :

$$
u(x)=\int_{0}^{\infty} e^{i \varrho^{k+1} x_{3}+\left(\sqrt{k} x_{2}-1\right) \varrho^{(k+1) / 2}-\frac{1}{k+1}\left(\varrho x_{1}\right)^{k+1}} d \varrho .
$$

Poisson strata. To state a revised conjecture of Treves [Tr3] about a necessary and sufficient condition for the analytic hypoellipticity of $\Delta_{X}$ we need to introduce a certain stratification of the characteristic set. We define

$$
\begin{aligned}
& \Sigma_{1} \doteq \Sigma=\left\{X_{j}(x, \xi)=0: j=1, \ldots, \nu\right\}, \\
& \Sigma_{2} \doteq \Sigma_{1} \cap\left\{\left\{X_{i}, X_{j}\right\}(x, \xi)=0: i, j=1, \ldots, \nu\right\}, \\
& \Sigma_{3} \doteq \Sigma_{2} \cap\left\{\left\{X_{l},\left\{X_{i}, X_{j}\right\}\right\}(x, \xi)=0: l, i, j=1, \ldots, \nu\right\}, \ldots
\end{aligned}
$$

We recall that for two functions $f(x, \xi)$ and $g(x, \xi)$ defined in $T^{*} \Omega$ the Poisson bracket $\{\cdot, \cdot\}$ is defined by

$$
\{f, g\}=\sum_{j=1}^{n} \frac{\partial f}{\partial \xi_{j}} \frac{\partial g}{\partial x_{j}}-\frac{\partial f}{\partial x_{j}} \frac{\partial g}{\partial \xi_{j}} .
$$

The sets $\Sigma_{j}$ are called the Poisson strata defined by the symbols of the vector fields $X_{j}$. Since the bracket condition holds, only a finite number of the Poisson strata $\Sigma_{j}$ are non-empty.

EXAmPle 2.1. Consider the operator $P_{k}$ in $(2.5)$ when $k=3$. That is, we let

$$
\Delta_{X}=\left(\frac{\partial}{\partial x_{1}}\right)^{2}+\left(x_{1} \frac{\partial}{\partial x_{2}}\right)^{2}+\left(x_{1}^{3} \frac{\partial}{\partial x_{3}}\right)^{2} .
$$

In this case the symbols of the vector fields are

$$
X_{1}(x, \xi)=\xi_{1}, \quad X_{2}(x, \xi)=x_{1} \xi_{2}, \quad X_{3}(x, \xi)=x_{1}^{3} \xi_{3} .
$$

The first Poisson stratum is given by the characteristic set $\Sigma$. That is,

$$
\Sigma_{1}=\left\{x_{1}=\xi_{1}=0\right\} \subset T^{*} \mathbb{R}^{3}-0 .
$$

Since the non-zero brackets of length two are

$$
\left\{X_{1}, X_{2}\right\}=\xi_{2}, \quad\left\{X_{1}, X_{3}\right\}=3 x_{1}^{2} \xi_{3}
$$

the second Poisson stratum $\Sigma_{2}$ is

$$
\Sigma_{2}=\Sigma_{1} \cap\left\{\xi_{2}=3 x_{1}^{2} \xi_{3}=0\right\}=\left\{x_{1}=\xi_{1}=\xi_{2}=0, \xi_{3} \neq 0\right\} .
$$

Since the non-zero bracket of length three is $\left\{X_{1},\left\{X_{1}, X_{3}\right\}\right\}=6 x_{1} \xi_{3}$ we have

$$
\Sigma_{3}=\Sigma_{2} \cap\left\{6 x_{1} \xi_{3}=0\right\}=\Sigma_{2} .
$$

Finally, $\left\{X_{1},\left\{X_{1},\left\{X_{1}, X_{3}\right\}\right\}\right\}=6 \xi_{3}$, and since $\xi_{3} \neq 0$ on $\Sigma_{3}$ we have

$$
\Sigma_{4}=\emptyset=\Sigma_{5}=\Sigma_{6}=\ldots
$$


Observe that the first Poisson stratum is symplectic while $\Sigma_{2}$ and $\Sigma_{3}$ are not. This observation has led Treves [Tr3] to the following new conjecture.

Conjecture 2. A necessary and sufficient condition for $\Delta_{X}$ to be analytic hypoelliptic is that all Poisson strata defined by the symbols of the vector fields $X_{j}$ are symplectic.

We mention that Bove and Tartakoff in [BTa1] and [BTa2] have formulated a conjecture on the optimal Gevrey, $G^{s}$, regularity of $\Delta_{X}$ based on the Poisson strata $\Sigma_{j}$. We do not formulate it here. However, for our example above it reads as follows:

$$
\text { Best } s=\frac{\text { length of bracket needed to obtain } \partial_{x_{3}}}{\text { length of bracket needed to obtain } \partial_{x_{2}}}=\frac{4}{2}=2 \text {. }
$$

Observe that the singular solutions (2.13) constructed above have optimal regularity 2. For the more general operators (2.6) of Oleunik and Radkevich it has been shown in [Ch5] that $P$ is $G^{s}$ hypoelliptic if and only if $s \geq$ $(k+1) /(m+1)$. Thus the optimal exponent is $(k+1) /(m+1)$, which is equal to 2 in the case of the operators in (2.5).

For more results on the local analytic hypoellipticity for sums of squares of vector fields we refer the reader to the following incomplete list of works: Christ [Ch2], Derridj and Zuily [DZ], Grigis and Rothschild [GR], Grigis and Sjöstrand [GS], Hanges and Himonas [HH1], Helffer [He], Matsuzawa [M], Menikoff [Me], Métivier [Met2], and Pham The Lai and Robert [PR].

3. Global analytic hypoellipticity. Next we discuss the problem of global analytic hypoellipticity for the case where the manifold is a torus. Let $b$ be a real-valued and real-analytic function defined near $0 \in \mathbb{R}$. It was shown in [HH2] that the operator

$$
\partial_{t}^{2}+\partial_{x}^{2}+\left(b(t) \partial_{y}\right)^{2}
$$

is analytic hypoelliptic near $0 \in \mathbb{R}^{3}$ if and only if $b(0) \neq 0$. By the results in [Tr1], [Ta1], and [Ch1] the operator

$$
\partial_{t}^{2}+\left(\partial_{x}+b(t) \partial_{y}\right)^{2}, \quad b(0)=0,
$$

is analytic hypoelliptic near $0 \in \mathbb{R}^{3}$ if and only if $b^{\prime}(0) \neq 0$. However, if $b$ is a real-valued function in $\mathcal{C}^{\omega}(\mathbb{T})$ then the first operator is globally analytic hypoelliptic in $\mathbb{T}^{3}$ if and only if $b$ is not identically zero, and the second operator is globally analytic hypoelliptic in $\mathbb{T}^{3}$ if and only if $b^{\prime}$ is not identically zero. In both cases the condition is equivalent to the bracket condition in $\mathbb{T}^{3}$. Thus these operators provide examples where global analytic hypoellipticity holds under the bracket condition and local analytic hypoellipticity fails. The global analytic hypoellipticity of these operators follows from the following result in Cordaro-Himonas [CH2]. 
Theorem 3.1. Consider the torus $\mathbb{T}^{N}=\mathbb{T}^{m} \times \mathbb{T}^{n}$ with variables $(x, t)$, $x=\left(x_{1}, \ldots, x_{m}\right), t=\left(t_{1}, \ldots, t_{n}\right)$, and let

$$
X_{j}=\sum_{k=1}^{n} a_{j k}(t) \frac{\partial}{\partial t_{k}}+\sum_{k=1}^{m} b_{j_{k}}(t) \frac{\partial}{\partial x_{j}}, \quad j=0, \ldots, \nu,
$$

be real vector fields with coefficients in $\mathcal{C}^{\omega}\left(\mathbb{T}^{n}\right)$, and $c=c(x, t) \in \mathcal{C}^{\omega}\left(\mathbb{T}^{m+n}\right)$ be complex-valued. Suppose the following two conditions hold:

(i) Every point of $\mathbb{T}^{m+n}$ is of finite type.

(ii) The vector fields $\sum_{k=1}^{n} a_{j k}(t) \partial / \partial t_{k}, j=1, \ldots, \nu$, span $T_{t}\left(\mathbb{T}^{n}\right)$ for every $t \in \mathbb{T}^{n}$.

Then the operator

$$
P=\sum_{j=1}^{\nu} X_{j}^{2}+X_{0}+c
$$

is globally analytic hypoelliptic in $\mathbb{T}^{N}$.

A generalization of Theorem 3.1 was obtained by Christ [Ch3] under the assumption of a certain symmetry condition, which does not hold here because of the dependence of $c$ on $x$. A different generalization has been proved by Tartakoff [Ta3] under the restriction $\nu=n$, but with $P$ in a more general form and assumed to satisfy a maximal estimate. Also, we mention the related work of Chen [C], Komatsu [Ko], Derridj-Tartakoff [DT], [Ta2], and [CH1]. Theorem 3.1 is only a partial result on the problem of global analytic hypoellipticity. It is far from clear what is a necessary and sufficient condition for the global analytic hypoellipticity of a sum of squares operator on a torus.

Open Problem 2. On a torus, and more generally on an analytic manifold, find necessary and sufficient conditions for the global analytic hypoellipticity of the sum of squares operator.

We mention that the bracket condition is not sufficient for global analytic hypoellipticity (see [Ch4]). It is not necessary either. This follows from the following generalization of operator (3.2). It also provides some insight into the kind of conditions needed for global analytic hypoellipticity.

TheOREm 3.2. Let $a, b$ in $\mathcal{C}^{\omega}(\mathbb{T})$ be real-valued. Then the operator

$$
P=-\partial_{t}^{2}-\left(a(t) \partial_{x}+b(t) \partial_{y}\right)^{2}
$$

is globally analytic hypoelliptic in $\mathbb{T}^{3}$ if and only if a is not identically zero and $b \neq \lambda a$ for any $\lambda \in \mathbb{Q} \cup \mathbb{L}_{e}$ where $\mathbb{Q}$ are the rationals and $\mathbb{L}_{e}$ are the exponentially Liouville numbers. 
We recall that an irrational number $\lambda$ is exponentially Liouville if there is an $\varepsilon_{0}>0$ such that

$$
|\lambda-p / q| \leq e^{-\varepsilon_{0} q} \quad \text { for infinitely many }(p, q) \in \mathbb{Z} \times \mathbb{N} .
$$

Equivalently, $\lambda$ is not exponentially Liouville if for any $\varepsilon>0$ there is $C_{\varepsilon}>0$ such that

$$
|\lambda-p / q| \geq C_{\varepsilon} e^{-\varepsilon q} \quad \text { for all }(p, q) \in \mathbb{Z} \times \mathbb{N} .
$$

Observe that (3.5) obviously holds for $\lambda \in \mathbb{Q}$. Also, we recall that $u \in$ $\mathcal{D}^{\prime}\left(\mathbb{T}^{n}\right)$, the space of distributions, is analytic in $\mathbb{T}^{n}$ if and only if its Fourier transform (coefficients) satisfies the estimate

$$
|\widehat{u}(\xi)| \leq c e^{-\varepsilon|\xi|}, \quad \xi \in \mathbb{Z}^{n},
$$

for some $\varepsilon>0$ and $c>0$.

Proof of Theorem 3.2. If $a=0$ then $P$ in (3.4) is not globally analytic hypoelliptic since any function $u=u(x)$ is a solution to $P u=0$. If $a \neq 0$ and $b=\lambda a$ for some $\lambda \in \mathbb{Q} \cup \mathbb{L}_{e}$ then $P$ takes the form $P=-\partial_{t}^{2}-a(t)^{2} L^{2}$, where $L=\partial_{x}+\lambda \partial_{y}$. Since $\lambda \in \mathbb{Q} \cup \mathbb{L}_{e}$ by (3.5) there exists a sequence $\left(\xi_{j}, \eta_{j}\right) \in \mathbb{Z} \times \mathbb{N}$ with $\eta_{j} \rightarrow \infty$ such that

$$
\left|L\left(\xi_{j}, \eta_{j}\right)\right|=\left|\xi_{j}+\lambda \eta_{j}\right|=\left|\eta_{j}\right|\left|\xi_{j} / \eta_{j}+\lambda\right| \leq c_{0} e^{-\varepsilon_{0} \eta_{j}} .
$$

If we define

$$
u(x, y)=\sum_{j=1}^{\infty} e^{i\left(x \xi_{j}+y \eta_{j}\right)},
$$

then $u \in \mathcal{D}^{\prime}\left(\mathbb{T}^{2}\right)-\mathcal{C}^{\omega}\left(\mathbb{T}^{2}\right)$ and

$$
L u(x, y)=\sum_{j=1}^{\infty} i L\left(\xi_{j}, \eta_{j}\right) e^{i\left(x \xi_{j}+y \eta_{j}\right)} .
$$

By (3.7) we can find $J \in \mathbb{N}$ such that if $j \geq J$ then $\left|\left(\xi_{j}, \eta_{j}\right)\right| \leq c \eta_{j}$ for some $c>0$. This together with (3.7) gives

$$
\left|L\left(\xi_{j}, \eta_{j}\right)\right| \leq c_{0}^{\prime} e^{-\varepsilon_{0}\left|\left(\xi_{j}, \eta_{j}\right)\right|} \quad \text { for all } j \in \mathbb{N},
$$

which implies that $L u \in \mathcal{C}^{\omega}\left(\mathbb{T}^{2}\right)$. Since $P u=-a(t)^{2} L(L u)$ we see that $P u$ is analytic in $\mathbb{T}^{3}$ while $u$ is not analytic. Therefore $P$ is not globally analytic hypoelliptic. This part of the proof was along the lines of the work of Greenfield and Wallach [GW].

Conversely, assume that $a \neq 0$ and $b \neq \lambda a$ for all $\lambda \in \mathbb{Q} \cup \mathbb{L}_{e}$. Let $u \in \mathcal{D}^{\prime}\left(\mathbb{T}^{3}\right)$ and $f \in \mathcal{C}^{\omega}\left(\mathbb{T}^{3}\right)$ be such that

$$
P u=f .
$$


We need to show that $u \in \mathcal{C}^{\omega}\left(\mathbb{T}^{3}\right)$. For this we take partial Fourier transform with respect to $(x, y)$ and obtain

$$
-\widehat{u}_{t t}(t, \xi, \eta)+(a(t) \xi+b(t) \eta)^{2} \widehat{u}(t, \xi, \eta)=\widehat{f}(t, \xi, \eta) .
$$

Since equation (3.11) is elliptic in $t$ we have $\widehat{u}(\cdot, \xi, \eta) \in \mathcal{C}^{\omega}(\mathbb{T})$. Multiplying by $\bar{u}(t, \xi, \eta)$ and integrating by parts with respect to $t$ gives

$$
\|\widehat{u}(\cdot, \xi, \eta)\|_{w}^{2}=\int_{\mathbb{T}} \widehat{f}(t, \xi, \eta) \bar{u}(t, \xi, \eta) d t,
$$

where for $\varphi \in \mathcal{C}^{1}(\mathbb{T})$ we define

$$
\|\varphi\|_{w}^{2}=\left\|\varphi^{\prime}\right\|_{L^{2}(\mathbb{T})}^{2}+\int_{\mathbb{T}} w^{2}(t, \xi, \eta)|\varphi(t)|^{2} d t \quad \text { with } w=a(t) \xi+b(t) \eta .
$$

If $b=\lambda a$ for some $\lambda \in \mathbb{R}-\left(\mathbb{Q} \cup \mathbb{L}_{e}\right)$ then $w^{2}(t, \xi, \eta)=a^{2}(t)(\xi+\lambda \eta)^{2}$. If $\eta=0$ then $w^{2} \geq a^{2}(t) \xi^{2} \geq a^{2}(t)$ for $\xi \neq 0$. For $\eta \neq 0$ we have

$$
w^{2}(t, \xi, \eta)=a^{2}(t) \eta^{2}\left(\frac{\xi}{\eta}+\lambda\right)^{2} \geq a^{2}(t) C_{\varepsilon} e^{-\varepsilon|\eta|} \quad \text { for any } \varepsilon>0 .
$$

Since $a \neq 0$ there is an open interval of positive length $\delta=\delta(a)$, and a constant $\alpha_{1}=\alpha_{1}(a)$ such that for any $\varepsilon>0$,

$$
w^{2}(t, \xi, \eta) \geq \alpha_{1} C_{\varepsilon} e^{-\varepsilon|(\xi, \eta)|}, \quad t \in I,(\xi, \eta) \in \mathbb{Z}^{2}-0,
$$

for some $C_{\varepsilon}>0$ depending on $\varepsilon$.

If $b \neq \lambda a$ for all $\lambda \in \mathbb{R}$ then there is $t_{0} \in(-\pi, \pi)$ such that $(b / a)^{\prime}\left(t_{0}\right) \neq 0$ for all $t$ near $t_{0}$. In this case we can find a $\delta>0$ depending only on $(a, b)$ such that for each $(\xi, \eta) \neq 0$ there is an open interval $I=I(\xi, \eta)$ of length $\delta$ and

$$
w^{2}(t, \xi, \eta) \geq \alpha_{2}, \quad t \in I,
$$

where $\alpha_{2}>0$ is independent of $(\xi, \eta)$.

By the fundamental theorem of calculus, the Cauchy-Schwarz inequality, and integration for $t \in(-\pi, \pi)$ and $s \in I$, we obtain

$$
\|\varphi\|_{L^{2}(\mathbb{T})}^{2} \leq c\left(\left\|\varphi^{\prime}\right\|_{L^{2}(\mathbb{T})}^{2}+\int_{I}|\varphi(s)|^{2} d s\right) .
$$

Moreover, by (3.14) and (3.15) we have

$$
\int_{I}|\varphi(s)|^{2} d s \leq \alpha C_{\varepsilon}^{\prime} e^{\varepsilon|(\xi, \eta)|} \int_{\mathbb{T}} w^{2}(t, \xi, \eta)|\varphi(s)|^{2} d s,
$$

for some $\alpha>0$ depending on $(a, b)$, and $C_{\varepsilon}^{\prime}>0$ depending on $\varepsilon$. By (3.16) and (3.17) we obtain

$$
\|\varphi\|_{L^{2}(\mathbb{T})}^{2} \leq c_{1} C_{\varepsilon}^{\prime} e^{\varepsilon|(\xi, \eta)|}\|\varphi\|_{w}^{2} .
$$


Finally, (3.18) applied with $\varphi(t)=\widehat{u}(t, \xi, \eta),(3.12)$, and the Cauchy-Schwarz inequality give

$$
\begin{aligned}
& \|\widehat{u}(\cdot, \xi, \eta)\|_{L^{2}(\mathbb{T})} \\
& \quad \leq c C_{\varepsilon}^{\prime} e^{\varepsilon|(\xi, \eta)|}\|\widehat{f}(\cdot, \xi, \eta)\|_{L^{2}(\mathbb{T})} \quad \text { for all }(\xi, \eta) \in \mathbb{Z}^{2}-0 .
\end{aligned}
$$

Since $f$ is analytic there is $\varepsilon_{0}>0$ such that

$$
\|\widehat{f}(\cdot, \xi, \eta)\|_{L^{2}(\mathbb{T})} \leq c_{0} e^{-\varepsilon_{0}|(\xi, \eta)|} .
$$

If we choose $\varepsilon=\varepsilon_{1}=\varepsilon_{0} / 2$ then (3.19) and (3.20) give

$$
\|\widehat{u}(\cdot, \xi, \eta)\|_{L^{2}(\mathbb{T})} \leq c_{1} e^{-\varepsilon_{1}|(\xi, \eta)|} .
$$

Then (3.21) and the Cauchy-Schwarz inequality give

$$
|\widehat{u}(\tau, \xi, \eta)| \leq c_{2} e^{-\varepsilon_{1}|(\xi, \eta)|}, \quad(\tau, \xi, \eta) \in \mathbb{Z}^{3}-0,(\xi, \eta) \neq(0,0) .
$$

Now let $\left(\tau_{0}, \xi_{0}, \eta_{0}\right)$ with $\left(\xi_{0}, \eta_{0}\right) \neq(0,0)$. If we choose

$$
m_{0}=2 \max \left\{\tau_{0} /\left|\left(\xi_{0}, \eta_{0}\right)\right|, 1\right\},
$$

then the cone $\Gamma_{0}=\left\{(\tau, \xi, \eta):|\tau|<m_{0}|(\xi, \eta)|\right\}$ is a conic neighborhood of $\left(\tau_{0}, \xi_{0}, \eta_{0}\right)$, and $(3.22)$ gives

$$
|\widehat{u}(\tau, \xi, \eta)| \leq c_{2} e^{-\varepsilon_{2}|(\tau, \xi, \eta)|}, \quad(\tau, \xi, \eta) \in \Gamma_{0},
$$

for some $\varepsilon_{2}>0$. By microlocal elliptic theory, estimates similar to (3.23) are also valid near each elliptic point $\left(\tau_{0}, 0,0\right)$. Thus $u$ is analytic in $\mathbb{T}^{3}$. This completes the proof of Theorem 3.2.

4. Concluding remark. The general sum of squares operator is of the form $\Delta_{X}+X_{0}+c$. If $X_{0}$ is a complex vector field then additional phenomena may appear in both local and global analytic hypoellipticity. For example the Grushin operator $\partial_{t}^{2}+\left(t \partial_{x}\right)^{2}+i(\mu+1) \partial_{x}$ is hypoelliptic in $\mathbb{R}^{2}$ if and only if $\mu \neq 2 j, j=0,1, \ldots$; if $\mu=0$ then this operator takes the form $\bar{L} L$ with $L=\partial_{t}+i t \partial_{x}$, and one can easily construct singular solutions to $L u=0$. For this type of phenomena we refer the reader to Grushin [Gr], Boutet de Monvel and Treves [BT], Treves [T2], Gilioli [G], Gilioli and Treves [GT], and Hanges and Himonas [HH3].

For the global problem consider the operator $P=\bar{L} L+c$, where $L$ is a vector field in $\mathbb{T}^{2}$ of the form $L=\partial_{t}+i b(t) \partial_{x}$, with $b(t)$ a real-analytic and real-valued function in $\mathbb{T}$. It was shown in [CH2] that if all zeros of $b$ are of odd order and if $c \neq 0$ then $P$ is globally analytic hypoelliptic. Conversely, if $b$ has a zero of odd order and if $c=0$ then $P$ is not globally analytic hypoelliptic. Similar results for other operators have been obtained by Stein $[\mathrm{St}]$ and Kwon $[\mathrm{Kw}]$. 


\section{References}

[BG] M. S. Baouendi and C. Goulaouic, Nonanalytic-hypoellipticity for some degenerate elliptic operators, Bull. Amer. Math. Soc. 78 (1972), 483-486.

[BM] D. R. Bell and S. A. Mohammed, An extension of Hörmander's theorem for infinitely degenerate second-order operators, Duke Math. J. 78 (1995), 453-475.

[BT] L. Boutet de Monvel and F. Treves, On a class of pseudo-differential operators with double characteristics, Invent. Math. 24 (1974), 1-34.

[BTa1] A. Bove and D. S. Tartakoff, Optimal non-isotropic Gevrey exponents for sums of squares of vector fields, Comm. Partial Differential Equations 22 (1997), 1263-1282.

[BTa2] -, 一, On a conjecture of Treves: Analytic hypoellipticity and Poisson strata, preprint, 1997.

[C] S. C. Chen, Global analytic hypoellipticity of the $\bar{\partial}-$ Neumann problem on circular domains, Invent. Math. 92 (1988), 173-185.

[Ch1] M. Christ, A class of hypoelliptic PDE admitting nonanalytic solutions, in: Contemp. Math. 137, Amer. Math. Soc., 1992, 155-167.

[Ch2] -, A necessary condition for analytic hypoellipticity, Math. Res. Lett. 1 (1994), 241-248.

[Ch3] -, Global analytic hypoellipticity in the presence of symmetry, ibid., 559-563.

[Ch4] -, A progress report on analytic hypoellipticity, in: Geometric Complex Analysis, J. Noguchi (ed.), World Sci., 1996, 123-146.

[Ch5] - Intermediate optimal Gevrey exponents occur, Comm. Partial Differential Equations 22 (1997), 359-379.

[CH1] P. D. Cordaro and A. A. Himonas, Global analytic hypoellipticity for a class of degenerate elliptic operators on the torus, Math. Res. Lett. 1 (1994), 501-510.

[CH2] - - - Global analytic hypoellipticity for sums of squares of vector fields, Trans. Amer. Math. Soc., to appear.

[D] M. Derridj, Un problème aux limites pour une classe d'opérateurs du second ordre hypoelliptiques, Ann. Inst. Fourier (Grenoble) 21 (1971), no. 4, 99-148.

[DT] M. Derridj and D. S. Tartakoff, Global analyticity for $\square_{b}$ on three dimensional CR manifolds, Comm. Partial Differential Equations 18 (1993), 18471868.

[DZ] M. Derridj et C. Zuily, Régularité analytique et Gevrey d'opérateurs elliptiques dégénérés, J. Math. Pures Appl. 52 (1973), 65-80.

[F] V. S. Fediŭ, Estimates in $H_{(s)}$ norms and hypoellipticity, Dokl. Akad. Nauk SSSR 193 (1970), 301-303 (in Russian).

[G] A. Gilioli, A class of second-order evolution equations with double characteristics, Ann. Scuola Norm. Sup. Pisa Cl. Sci. (4) 3 (1976), 187-229.

[GT] A. Gilioli and F. Treves, An example in the solvability theory of linear PDE's, Amer. J. Math. 96 (1974), 367-385.

[GR] A. Grigis and L. P. Rothschild, A criterion for analytic hypoellipticity of a class of differential operators with polynomial coefficients, Ann. of Math. 118 (1983), 443-460.

[GS] A. Grigis et J. Sjöstrand, Front d'onde analytique et sommes de carrés de champs de vecteurs, Duke Math. J. 52 (1985), 35-51.

[GW] S. J. Greenfield and N. R. Wallach, Global hypoelliptic and Liouville numbers, Proc. Amer. Math. Soc. 31 (1972), 112-114.

[Gr] V. V. Grushin, On a class of hypoelliptic operators, Mat. Sb. 83 (1970), 456-473 (in Russian). 
[HH1] N. Hanges and A. A. Himonas, Singular solutions for sums of squares of vector fields, Comm. Partial Differential Equations 16 (1991), 1503-1511.

[HH2] - - - Analytic hypoellipticity for generalized Baouendi Goulaouic operators, J. Funct. Anal. 125 (1994), 309-325.

[HH3] -, - Singular solutions for a class of Grusin type operators, Proc. Amer. Math. Soc. 124 (1996), 1549-1557.

[HH4] - - - Non-analytic hypoellipticity in the presence of symplecticity, ibid. 126 (1998), 405-409.

[He] B. Helffer, Conditions nécessaires d'hypoanalyticité pour des opérateurs invariants à gauche homogènes sur un groupe nilpotent gradué, J. Differential Equations 44 (1982), 460-481.

[Ho] L. Hörmander, Hypoelliptic second order differential equations, Acta Math. 119 (1967), 147-171.

[K] J. J. Kohn, Pseudo-differential operators and hypoellipticity, in: Proc. Sympos. Pure Math. 23, Amer. Math. Soc., 1973, 61-70.

[Ko] G. Komatsu, Global analytic hypoellipticity of the $\bar{\partial}-$ Neumann problem, Tôhoku Math. J. 28 (1976), 145-156.

[KS] S. Kusuoka and D. Strook, Applications of the Malliavin calculus, Part II, J. Fac. Sci. Tokyo Sect. IA Math. 32 (1985), 1-76.

[Kw] K. H. Kwon, Concatenations applied to analytic hypoellipticity of operators with double characteristics, Trans. Amer. Math. Soc., 283 (1984), 753-763.

[M] T. Matsuzawa, Sur les équations $u_{t} t+t^{\alpha} u_{x} x=f(\alpha \geq 0)$, Nagoya Math. J. 42 (1971), 43-55.

[Me] A. Menikoff, Some examples of hypoelliptic partial differential equations, Math. Ann. 221 (1976), 167-181.

[Met1] G. Métivier, Analytic hypoellipticity for operators with multiple characteristics, Comm. Partial Differential Equations 1 (1981), 1-90.

[Met2] - Une classe d'opérateurs non hypoelliptiques analytiques, Indiana Univ. Math. J. 29 (1980), 823-860.

[OR1] O. A. Oleı̆nik and E. V. Radkevich, On the analyticity of solutions of linear differential equations and systems, Dokl. Akad. Nauk SSSR 207 (1972), 16141618 (in Russian).

[OR2] - - - Second Order Equations with Nonnegative Characteristic Form, Amer. Math. Soc. and Plenum Press, 1973.

[PR] Pham The Lai et D. Robert, Sur un problème aux valeurs propres non linéaire, Israel J. Math. 36 (1980), 169-186.

[RS] L. P. Rothschild and E. M. Stein, Hypoelliptic differential operators and nilpotent groups, Acta Math. 137 (1977), 247-320.

[S] J. Sjöstrand, Analytic wavefront sets and operators with multiple characteristics, Hokkaido Math. J. 12 (1983), 392-433.

[St] E. M. Stein, An example on the Heisenberg group related to the Lewy operator, Invent. Math. 69 (1982), 209-216.

[Ta1] D. S. Tartakoff, On the local real analyticity of solutions to $\square_{b}$ and the $\bar{\partial}$ Neumann problem, Acta Math. 145 (1980), 117-204.

[Ta2] - On the global real analyticity of solutions to $\square_{b}$ on compact manifolds, Comm. Partial Differential Equations 1 (1976), 283-311.

[Ta3] -, Global (and local) analyticity for second order orerators constructed from rigid vector fields on products of tori, Trans. Amer. Math. Soc. 348 (1996), 2577-2583. 
[Tr1] F. Treves, Analytic hypo-ellipticity of a class of pseudodifferential operators with double characteristics and applications to the $\bar{\partial}-$ Neumann problem, Comm. Partial Differential Equations 3 (1978), 475-642.

[Tr2] -, Concatenations of second-order evolution equations applied to local solvability and hypoellipticity, Comm. Pure Appl. Math. 26 (1973), 201-250.

[Tr3] —, Symplectic geometry and analytic hypo-ellipticity, preprint, 1996.

Department of Mathematics

University of Notre Dame

Notre Dame, Indiana 46556

U.S.A.

E-mail: alex.a.himonas.1@nd.edu

Reçu par la Rédaction le 5.1.1998

Révisé le 28.8.1998 